\title{
LA-UR-20-23577
}

Approved for public release; distribution is unlimited.

Title: $\quad$ Using Deep Neural Networks to Extract Fireball Parameters from Infrared Spectral Data

Author(s): $\quad$ Armstrong, Derek Elswick

Gorka, Joseph Gabriel

Intended for: Virtual meeting / symposium.

Issued: 
Disclaimer:

Los Alamos National Laboratory, an affirmative action/equal opportunity employer, is operated by Triad National Security, LLC for the National Nuclear Security Administration of U.S. Department of Energy under contract 89233218CNA000001. By approving this article, the publisher recognizes that the U.S. Government retains nonexclusive, royalty-free license to publish or reproduce the published form of this contribution, or to allow others to do so, for U.S. Government purposes. Los Alamos National Laboratory requests that the publisher identify this article as work performed under the auspices of the U.S. Department of Energy. Los Alamos National Laboratory strongly supports academic freedom and a researcher's right to publish; as an institution, however, the Laboratory does not endorse the viewpoint of a publication or guarantee its technical correctness. 


\section{Using Deep Neural Networks to Extract Fireball Parameters from Infrared Spectral Data}

\section{ASME 2020 Virtual V\&V Symposium VVS2020-8802}

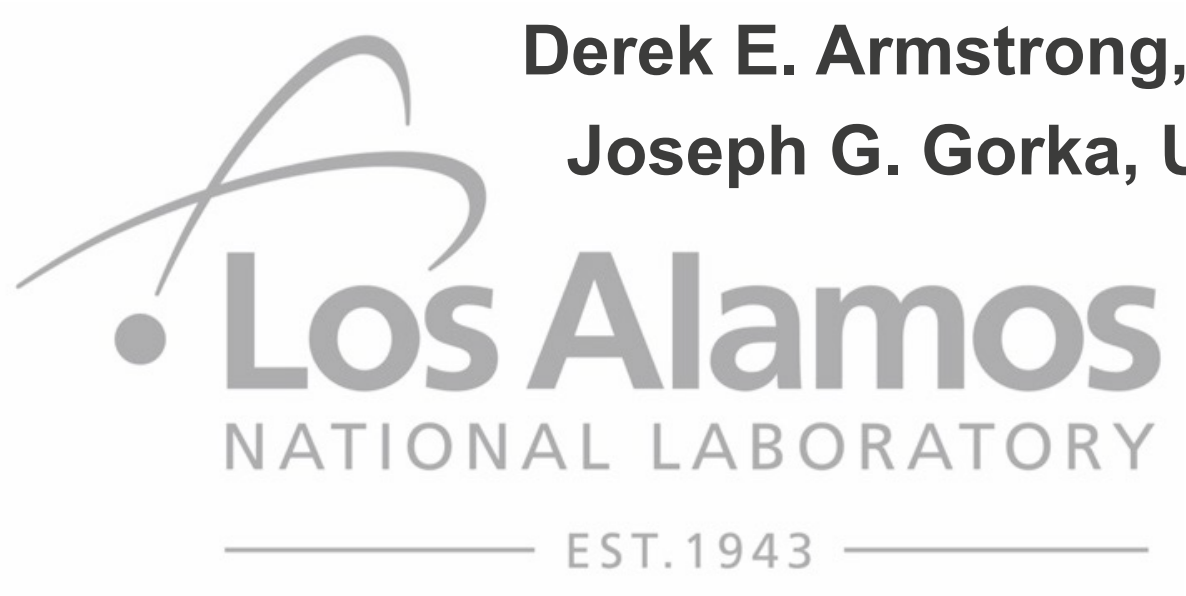

May 22, 2020 


\section{Overview}

\section{- Use spectrometers to monitor high explosive (HE) events in infrared region:}

-Spectrometers measure radiance in many (100s) of spectral bands.

-Sensors give information on the fireball such as temperature, size, soot quantity and gas species concentrations $\left(\mathrm{CO}, \mathrm{CO}_{2}, \mathrm{H}_{2} \mathrm{O}\right.$, etc.).
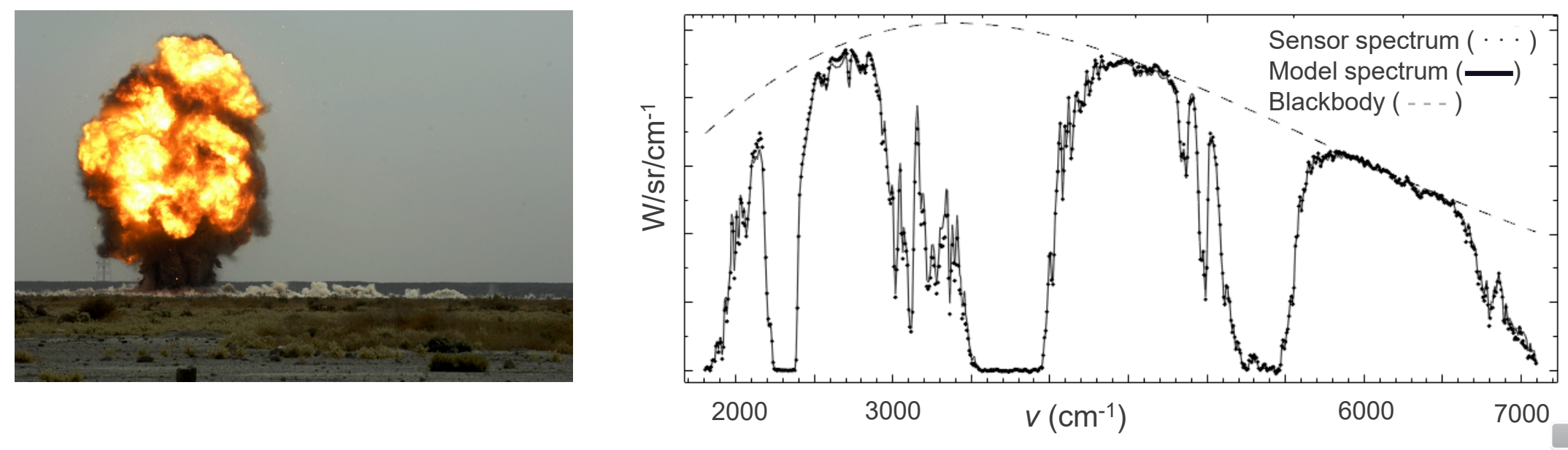


\section{Objective of Data Analysis}

- Develop methods to extract fireball information from remotely sensed infrared data with hundreds of spectral bands.

- Recent work looks at machine learning and deep neural networks.

- Validation of computational physics codes that simulate HE or similar events (equation-of-state (EOS), metal fragmentation, soot).

Image is $2 \mathrm{D}$ slice from $3 \mathrm{D}$ simulation

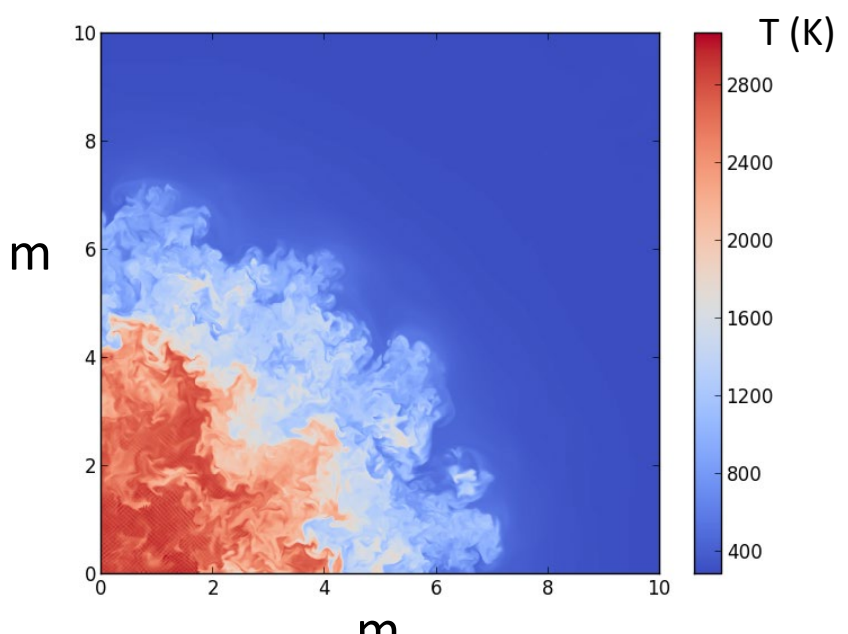

Models for gas species molar fractions in mixed HE/air zones

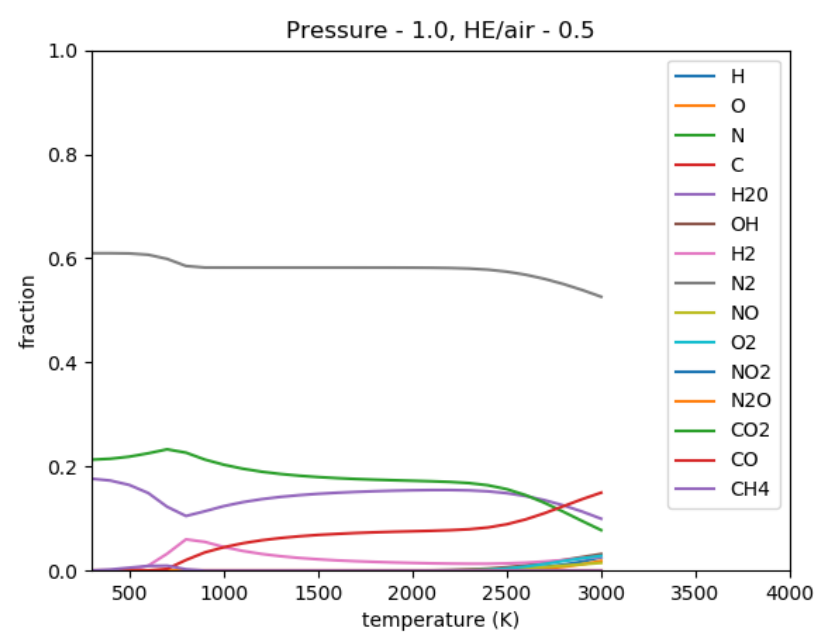




\section{Spectrometer Resolution and FTIR}

- Tradeoff between different resolutions (spatial vs temporal vs spectral):

- Sensors often sacrifice one type of resolution to improve the other two.

- This presentation considers specific FTIR spectrometer:

- FTIR (Fourier Transform Infrared): raw data is an interferogram.

-Single pixel, $\sim 16 \mathrm{~cm}^{-1}$ spectral resolution, $\sim 0.01 \mathrm{~s}$ temporal resolution.

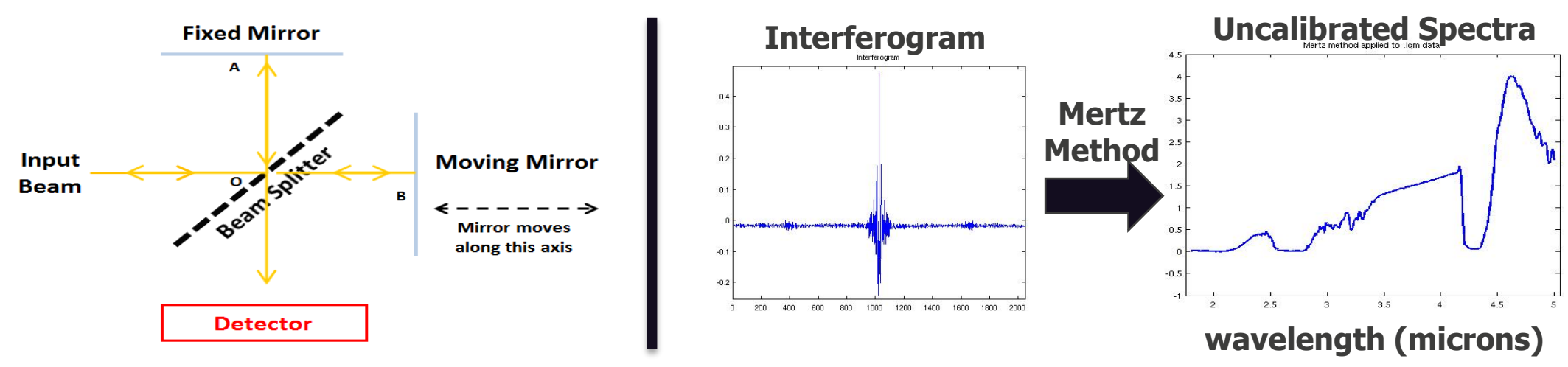




\section{Fireball Radiance Model}

At-sensor radiance model $R($.$) with gas$ and soot (no additional solid materials):

\section{Fit parameters in red}

$$
\begin{aligned}
& R(v)=l^{2} \varepsilon_{F B}(v) B\left(T_{F B}, v\right) \tau_{a t m}(v) \\
& =\underbrace{l^{2}}\left(1-e^{-l \kappa_{p}-l \sum \xi_{i} \sigma_{i}(v)}\right) \underbrace{B\left(T_{F B}, v\right) \underbrace{}_{a t m}(v)} \underbrace{\underbrace{}_{a t}} \\
& \text { area soot gases Blackbody Atmospheric } \\
& \text { function transmission }
\end{aligned}
$$

$T_{F B}$ is fireball temperature

$l^{2}$ is fireball area

$\kappa_{p}$ is soot absorption coefficient

$\sigma_{i}$ is gas cross sections

$\xi_{i}$ is gas concentrations in $\# / \mathrm{cm}^{3}$

Goal is to find fireball parameters in model $R($.$) that best fit data.$

Methods previously applied: Physics-based fitting, optimization, and Bayesian calibration. 


\section{Model for Fireball Radiance}

At sensor radiance given by: $R(v)=l^{2} \varepsilon_{F B}(v) B\left(T_{F B}, v\right) \tau_{a t m}(v)$

Red curve is blackbody

scaled by the fireball area.

Green curve is atmospheric transmission applied to blackbody.

Blue curve is FTIR.

Mismatch between green curve and data (blue) is due to fireball selective emission from fireball gas constituents.

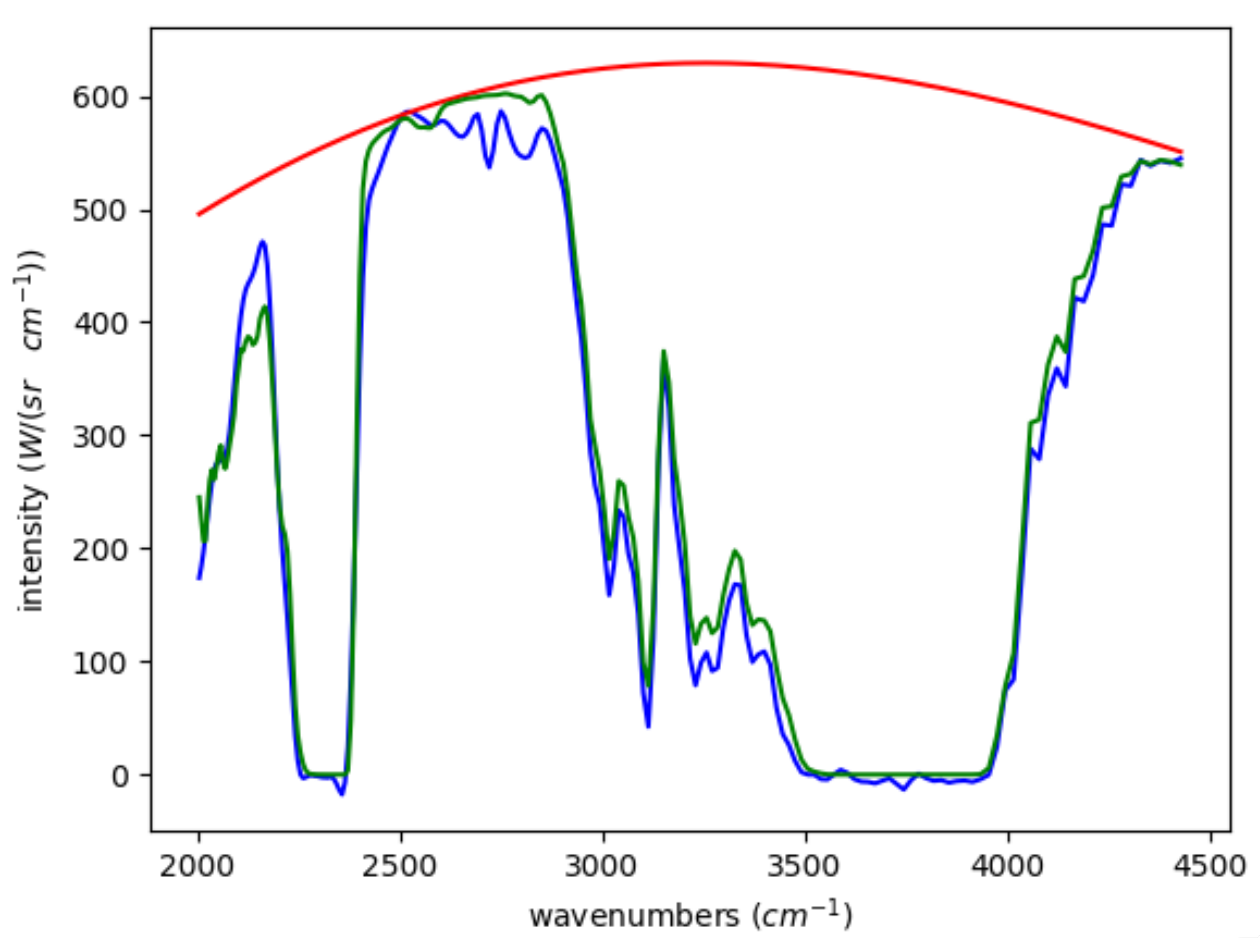




\section{Computational Challenges}

Spectral model is computed at Then convolved with a sensor high resolution: $0.001 \mathrm{~cm}^{-1}$

$$
\begin{aligned}
R(v) & =l^{2} \varepsilon_{F B}(v) B\left(T_{F B}, v\right) \tau_{a t m}(v) \quad R_{s} \\
& =l^{2}\left(1-e^{-l k_{p}-l \sum \xi_{i} \sigma_{i}(v)}\right) B\left(T_{F B}, v\right) \tau_{a t m}(v)
\end{aligned}
$$

$\mathrm{T}=1195 \mathrm{~K}, \quad l=330 \mathrm{~cm}$, soot $\left(\mathrm{cm}^{-1}\right)=0.001$, $\mathrm{XH} 2 \mathrm{O}\left(\# / \mathrm{cm}^{3}\right)=7.3 \mathrm{E} 17$, $X \mathrm{XO} 2\left(\# / \mathrm{cm}^{3}\right)=1.7 \mathrm{E} 18$, $\mathrm{XCO}\left(\# / \mathrm{cm}^{3}\right)=9.2 \mathrm{E} 16$.

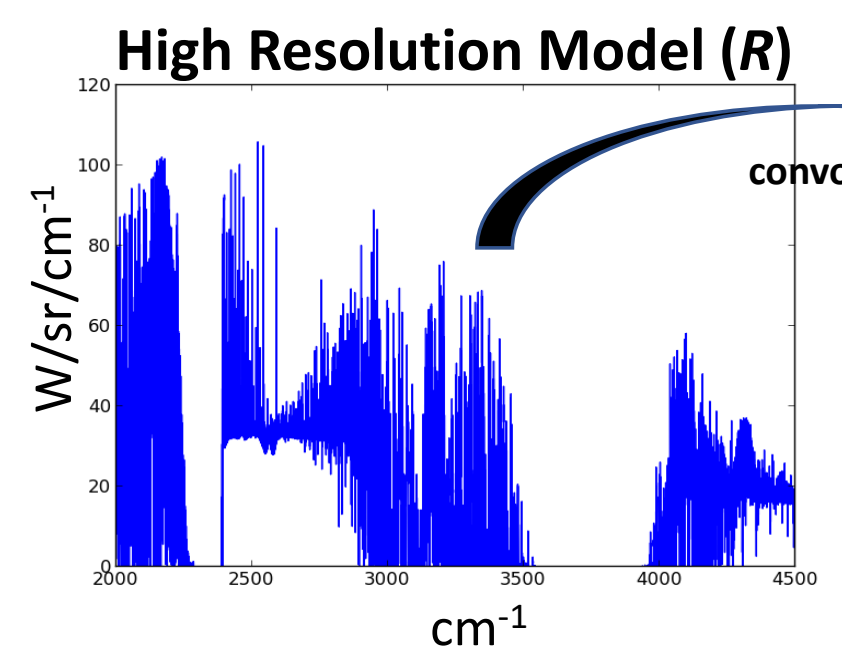

Instrument Line Shape (L)

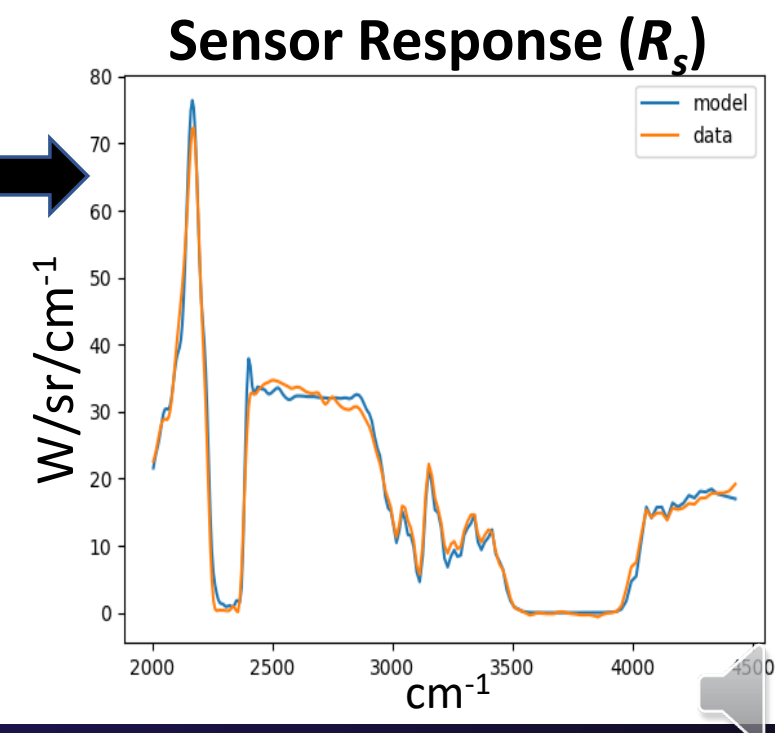




\section{Machine Learning}

- Applying machine learning to artificially generated spectra:

- Experimental data has no ground truth!

- Analysis of artificial data to evaluate the accuracy of methods.

- Deep and shallow learning applied to problem:

- Deep learning for full evaluation of regression accuracy.

- Shallow learning with physics information and determination of important spectral bands.

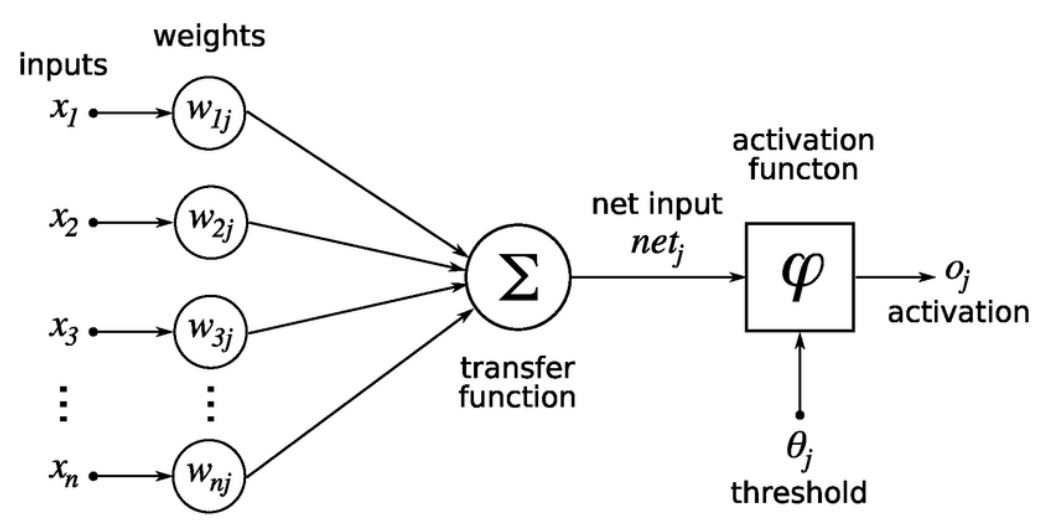




\section{Generation of Artificial Data Set}

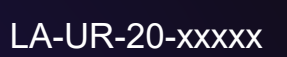

- Data with 388 spectral bands $\sim 1900$ to $5000 \mathrm{~cm}^{-1}$

- "Easy" data set of $\sim 400,000$ artificial spectra:

- Diameter kept constant at 3 meters.

- Additive noise at $\pm 0.5 \%$; narrow line-shape.

$-360,000$ for training \& testing; 36,000 for validation

- "Hard" data set of $\sim 400,000$ artificial spectra:

- All six parameters varied.

- Additive noise at $\pm 1 \%$; wide sensor line-shape.

\begin{tabular}{|c|c|c|}
\hline Parameter & $\begin{array}{c}\text { Lower } \\
\text { Bound }\end{array}$ & $\begin{array}{c}\text { Upper } \\
\text { Bound }\end{array}$ \\
\hline $\mathrm{T}(\mathrm{K})$ & 800 & 2200 \\
\hline diameter $(\mathrm{m})$ & 2 & 8 \\
\hline soot $\left(\mathrm{cm}^{-1}\right)$ & $1 \mathrm{E}-7$ & 0.04 \\
\hline $\mathrm{H} 2 \mathrm{O}\left(\# / \mathrm{cm}^{3}\right)$ & $1 \mathrm{E} 17$ & $1 \mathrm{E} 18$ \\
\hline $\mathrm{CO} 2\left(\# / \mathrm{cm}^{3}\right)$ & $1 \mathrm{E} 17$ & $2 \mathrm{E} 18$ \\
\hline $\mathrm{CO}\left(\# / \mathrm{cm}^{3}\right)$ & $1 \mathrm{E} 14$ & $1 \mathrm{E} 17$ \\
\hline
\end{tabular}

Data generated by sampling each parameter uniformly, except for soot.

Soot sampled such that the emissivity due to soot is uniform. 


\section{Deep Learning on Artificial Data}

Tested NN with many layers, including convolutional layers.

\begin{tabular}{|c|c|c|}
\hline \multicolumn{3}{|c|}{ Construction of Neural Network } \\
\hline Layer & Output Shape & Parameter Count \\
\hline 1D Convolution (Input) & $(193,16)$ & 64 \\
\hline 1D Convolution & $(96,32)$ & 1568 \\
\hline 1D Convolution & $(47,64)$ & 6208 \\
\hline 1D Convolution & $(23,128)$ & 24704 \\
\hline 1D Convolution & $(23,16)$ & 2064 \\
\hline Flatten & $($ None, 368) & 0 \\
\hline Dense & $($ None, 500) & 184500 \\
\hline Dense & $($ None, 250) & 125250 \\
\hline Dense & (None, 50) & 12550 \\
\hline Dense (Output) & (None, 5) & 255 \\
\hline
\end{tabular}

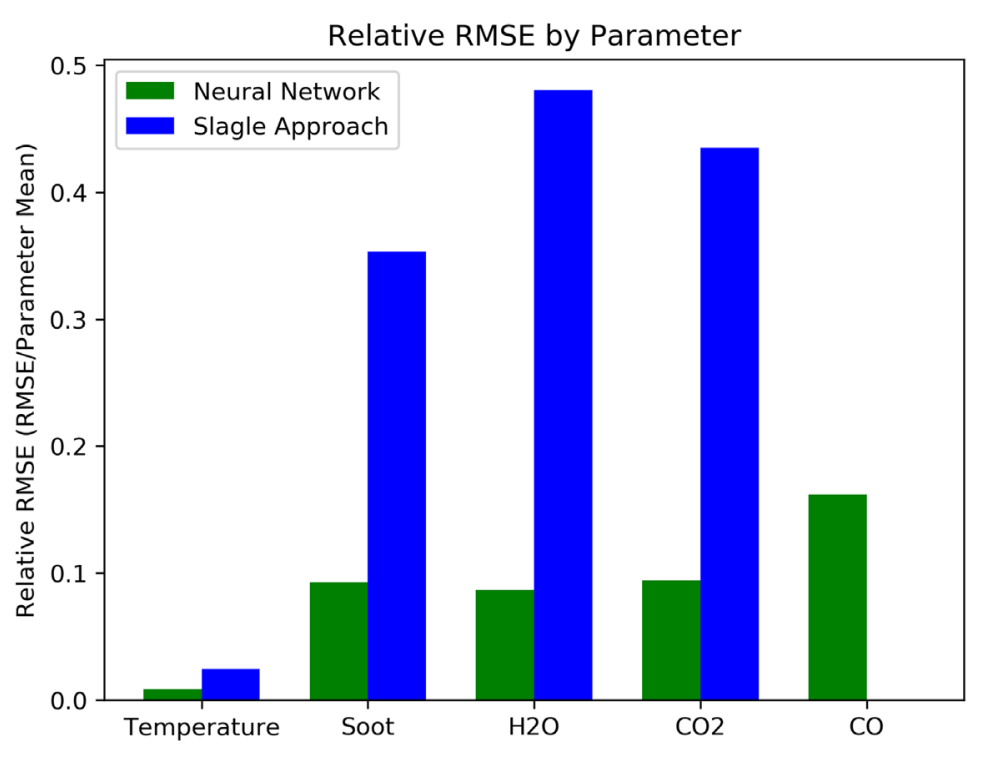

Compared results to Physics-based method of Slagle (AFIT thesis, 2009).

Fireball area kept constant to make problem easier ( $\mathrm{T}$, area, and soot are highly correlated) 


\section{Networks with Single Hidden Layer}

-What would the results be with a single hidden layer (HL)?

- How many nodes/neurons are necessary for a good model?

- Convolutional layers are counter-intuitive, especially for uncovering gases.

Fireball area kept constant.

$\mathrm{S} 1$ is RMSE/mean.

$\mathrm{S} 2$ is average relative error.

Validation statistics obtained from a set of 36000 artificial spectra.

Number of neurons for single $\mathrm{HL}$ varied from 128 to 2048 in a grid search.

\begin{tabular}{|c|c|c|}
\hline Parameter & $\begin{array}{c}\text { Deep NN } \\
\text { S1 }\end{array}$ & $\begin{array}{c}\text { Single HL } \\
\text { S1 / S2 }\end{array}$ \\
\hline $\mathrm{T}$ & 0.009 & $0.009 / 0.008$ \\
\hline soot & 0.093 & $0.070 / 0.091$ \\
\hline $\mathrm{H} 2 \mathrm{O}$ & 0.087 & $0.114 / 0.097$ \\
\hline $\mathrm{CO} 2$ & 0.094 & $0.110 / 0.111$ \\
\hline $\mathrm{CO}$ & 0.160 & $0.165 / 0.420$ \\
\hline
\end{tabular}




\section{Results with Varying Fireball Size}

- Temperature, fireball diameter, and soot quantity are highly correlated:

- All three impact magnitude of fireball radiance.

- Soot is a gray-body (emission is independent of wavenumber).

- Hard to include all three as unknown in the data.

Data 1: fireball size kept constant.

Data 2: all parameters vary.

Validation statistics obtained from a set of 36000 artificial spectra.

For soot and gases, output labels y transformed by $\log (y)$.

\begin{tabular}{|c|c|c|}
\hline $\begin{array}{c}\text { Para- } \\
\text { meter }\end{array}$ & $\begin{array}{c}\text { "Easy" Data 1 } \\
\text { S1 / S2 }\end{array}$ & $\begin{array}{c}\text { "Hard" Data 2 } \\
\text { S1 / S2 }\end{array}$ \\
\hline T & $0.009 / 0.008$ & $0.006 / 0.004$ \\
\hline diameter & NA & $0.012 / 0.009$ \\
\hline soot & $0.070 / 0.091$ & $0.240 / 0.050$ \\
\hline $\mathrm{H} 2 \mathrm{O}$ & $0.114 / 0.097$ & $0.203 / 0.141$ \\
\hline $\mathrm{CO} 2$ & $0.110 / 0.111$ & $0.286 / 0.199$ \\
\hline $\mathrm{CO}$ & $0.165 / 0.420$ & $0.522 / 0.656$ \\
\hline
\end{tabular}




\section{Are Better Results Possible?}

-Why do the gases become harder to estimate?

- Radiance is a smooth function of temperature, size, and soot.

- Would think that estimators could discern gases from non-smooth structure across bands.

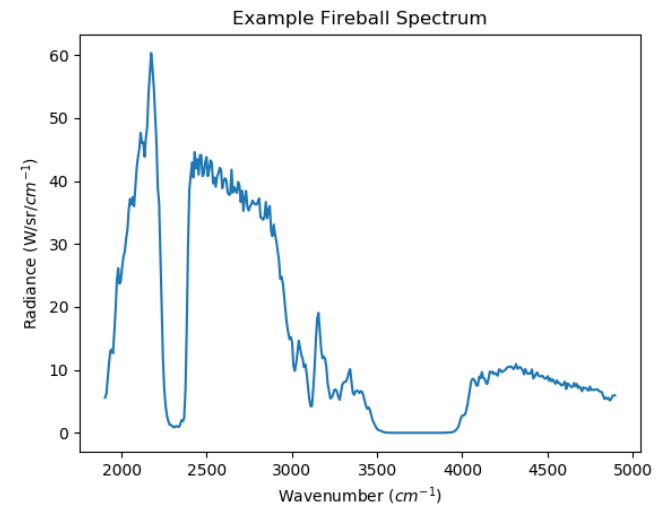

- Tested compensating data for temperature and size:

- Build estimator for $\mathrm{T}$ and size.

- Apply to a data set, divide out impact of $T$ and

$$
\frac{R(v)}{l^{2} B\left(T_{F B}, v\right)}=\varepsilon_{F B}(v) \tau_{a t m}(v)
$$
size, then fit to the other parameters.

THIS DID NOT WORK! RESULTS WERE WORSE! 


\section{Are the Results Misleading?}

\section{If soot concentration is large enough, estimates for the gases}

are unreliable:

- Soot makes the fireball opaque: $R(v)=l^{2}\left(1-e^{-l k_{p}-l \sum \xi_{i} \sigma_{i}(v)}\right) B\left(T_{F B}, v\right) \tau_{a t m}(v)$

- Should estimate emissivity of soot and if it's close to one, then it is likely that the gas estimates have high uncertainty.

Create a new label (output) equal to soot transmissivity $e^{-l \kappa_{p}}$ :

Training single HL network on this output resulted in RMSE of $\mathbf{0 . 0 3 9}$ on a validation set.

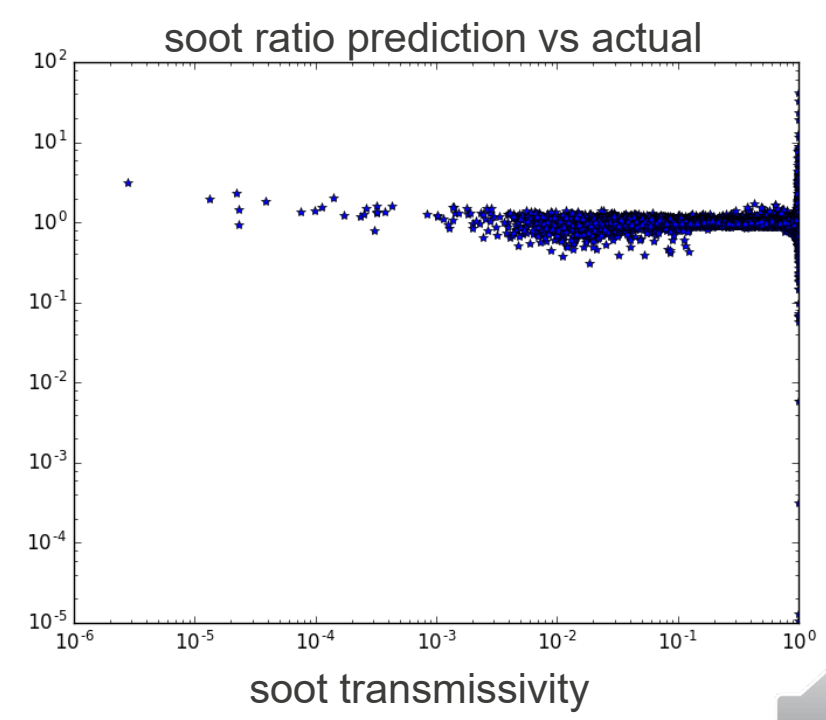




\section{Soot's Impact on T and Size}

\section{Results from a validation data set.}

T ratio prediction vs actual

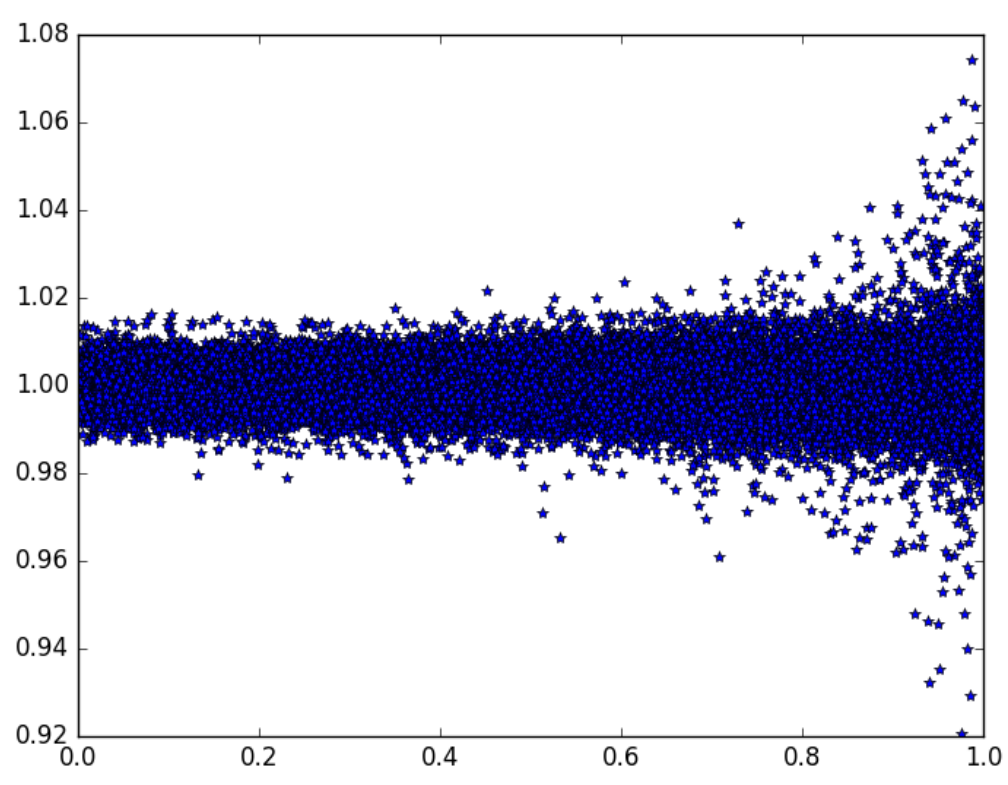

soot transmissivity diameter ratio prediction vs actual

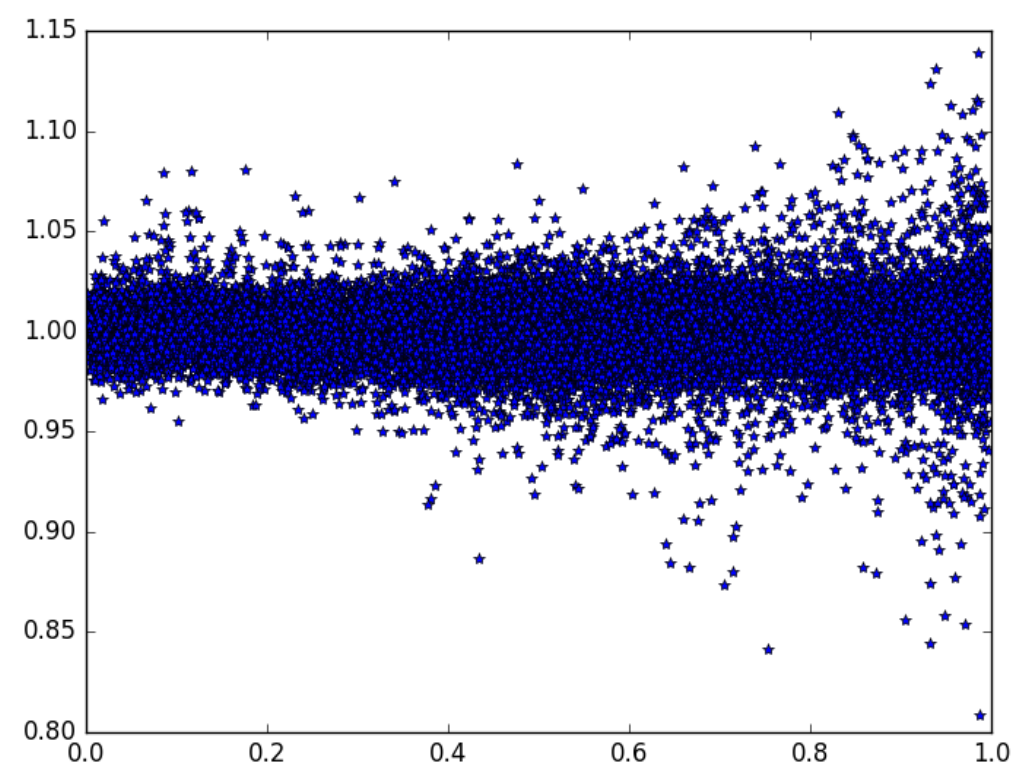

soot transmissivity 


\section{Soot's Impact on $\mathrm{H} 2 \mathrm{O} \& \mathrm{CO} 2$}

H2O ratio prediction vs actual

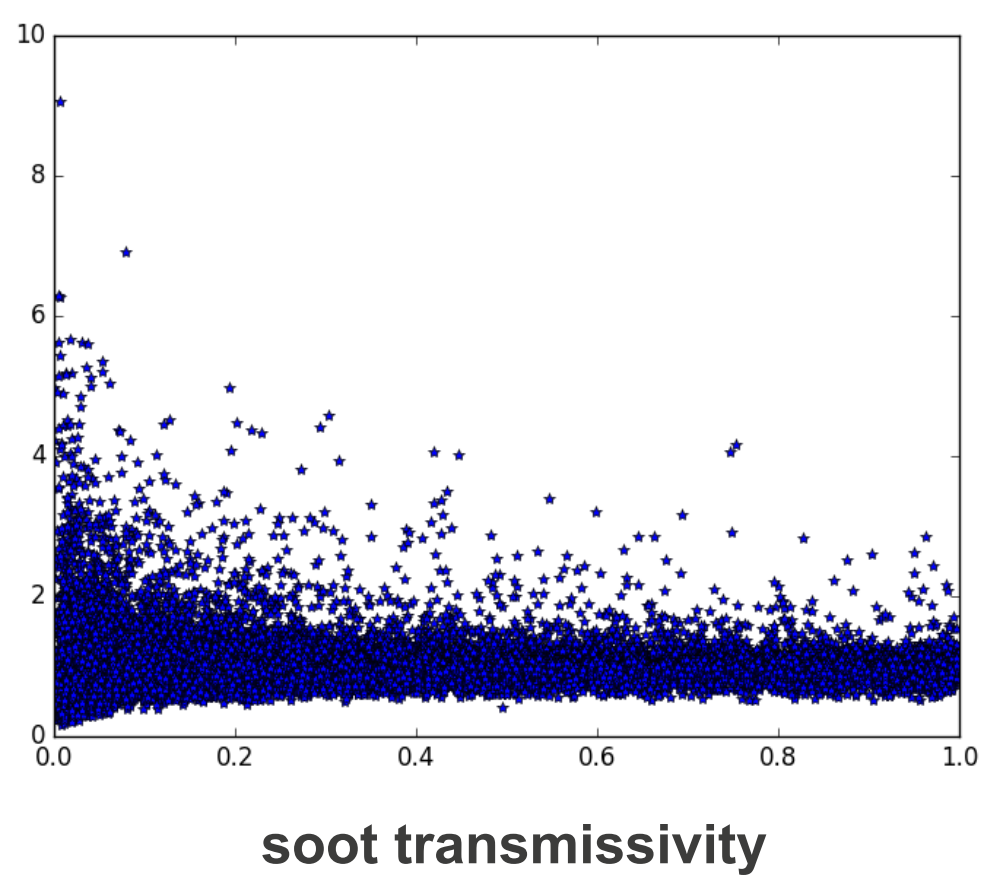

CO2 ratio prediction vs actual

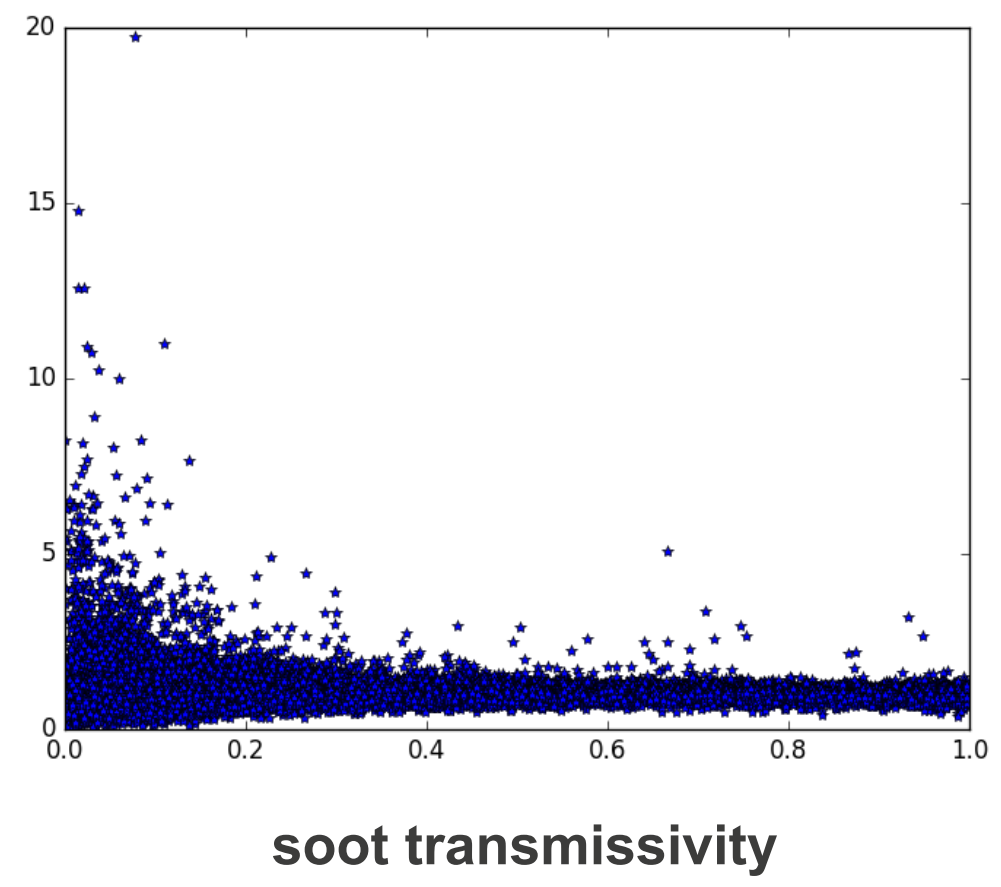




\section{Soot's Impact on CO}

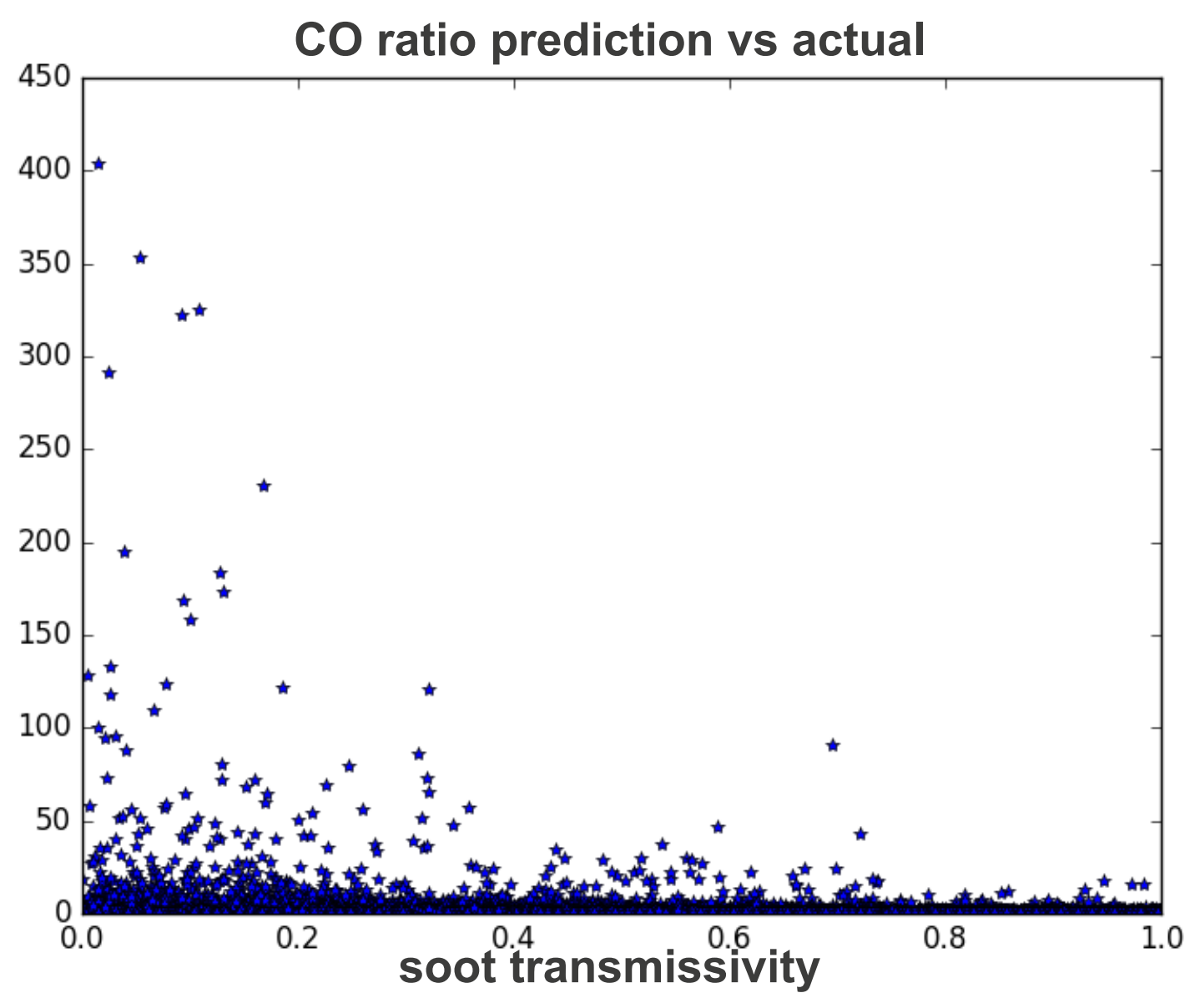




\section{Conclusion}

- Next Step (1): Examine outliers in prediction.

-Why is it difficult to predict the fireball parameters for some spectra?

- Next Step (2): Reverse fitting to uncover most "important bands"

- Train a network to predict spectral band given the six parameters.

- Bands that can be accurately predicted are "important".

- Analyzing artificial fireball spectra:

- Develop methods for recovering fireball parameters.

- Validation and improvement of computational physics codes. 